\title{
PREVALÊNCIA DE SOBREPESO E OBESIDADE DE CRIANÇAS E ADOLESCENTES NO BRASIL: UMA REVISÃO SISTEMÁTICA
}

\author{
Edilson Hobold ${ }^{1}$ \\ Miguel de Arruda $^{2}$
}

HOBOLD, E.; ARRUDA, M. de. Prevalência de sobrepeso e obesidade de crianças e adolescentes no Brasil: uma revisão sistemática. Arq. Cienc. Saúde UNIPAR, Umuarama, v. 18, n. 3, p, 189-197, set/dez. 2014.

\begin{abstract}
RESUMO: O objetivo deste estudo foi revisar as pesquisas de prevalência de sobrepeso e obesidade em crianças e adolescentes brasileiros. Foi realizado a busca eletrônica de artigos nas bases de dados Scielo, PubMed, MedLine e LILACS e na Biblioteca Eletrônica Bireme. Para efeito deste estudo, foram selecionadas as publicações de 2003 a 2013. Foram analisados 59 estudos que atenderam aos critérios de inclusão estabelecidos pelos autores. A maioria dos estudos foram desenvolvidos nas regiões Sul e Sudeste (71,2\%), com uma amostragem bem diversificada que variou de 328 a 79,6795 sujeitos. O Índice de Massa Corporal - IMC foi o protocolo mais utilizado (93,2\%) e o critério de classificação de sobrepeso/obesidade sugerido pela International Obesity Task Force - IOTF foi amplamente utilizado nos estudos (52,5\%). A prevalência de sobrepeso/obesidade oscilou de 7,4\% a 36,5\%, com mais de $70 \%$ dos estudos com prevalência de sobrepeso/ obesidade superior a $20 \%$, sendo mais elevada nos rapazes. Apesar das diferenças metodológicas, a maioria dos estudos com crianças e adolescentes brasileiros apresentou valores bastante elevados de prevalência de sobrepeso/obesidade sugerindo um constante crescimento deste problema em nosso país.
\end{abstract}

PALAVRAS-CHAVES: Sobrepeso; Obesidade; Crianças; Adolescentes; Revisão Sistemática.

\section{PREVALENCE OF OVERWEIGHT AND OBESITY IN CHILDREN AND ADOLESCENTS IN BRAZIL: A SYSTEMATIC REVIEW}

\begin{abstract}
The aim of this study was to review the research into the prevalence of overweight/obesity among Brazilian children and adolescents. An electronic search was conducted for articles in the Scielo, PubMed, MedLine and LILACS and Bireme Electronic Library databases. For this study, publications from 2003 to 2013 were selected. A total of 59 studies that met the inclusion criteria set by the authors were analyzed. Most of the studies were performed in the South and Southeast (71.2\%) regions, with a well diversified sampling ranging from 328 to 79,795 subjects. The Body Mass Index - BMI was the most frequently used protocol $(93.2 \%)$ and the criterion for overweight/obesity classification suggested by the International Obesity Task Force - IOTF was widely used in the studies (52.5\%). The overweight/obesity prevalence ranged from $7.4 \%$ to $36.5 \%$, with more than $70 \%$ of studies with overweight/obesity prevalence greater than $20 \%$, being higher in boys. Despite the methodological differences, most studies on Brazilian children and adolescents presented very high values of overweight/ obesity prevalence, suggesting constant growth of this issue in our country.

KEYWORDS: Overweight; Obesity; Children; Adolescents; Systematic review.
\end{abstract}

\section{Introdução}

O sobrepeso e a obesidade se tornariam nas últimas décadas, um grave problema de saúde pública observado na maioria dos países desenvolvidos e em desenvolvimento. Por si só, o sobrepeso e a obesidade não seriam tão preocupantes, mas sim os problemas que surgem a partir do excesso de gordura corporal, principalmente a visceral que é associada ao desenvolvimento de doenças crônicas, como diabetes, dislipidemias e hipertensão arterial.

A obesidade é definida como o acúmulo excessivo de gordura corporal, sob a forma de tecido adiposo, sendo consequência de balanço energético positivo, capaz de acarretar prejuízos à saúde dos indivíduos (WORLD HEALTH ORGANIZATION, 2000).

A cada pesquisa desenvolvida os índices se tornam mais alarmantes e, no Brasil a situação não é muito diferente. Especialmente nos últimos 30 anos o excesso de peso vem aumentando de forma alarmante. O Estudo Nacional de Despesa Familiar (ENDEF) desenvolvido em 1975 mostrou que os homens apresentavam 18,6\% de excesso de peso e as mulheres, 28,6\%. Após 28 anos a Pesquisa de Orçamentos Familiares (POF), realizada em 2003, indicou um aumento bem significativo dessa prevalência, atingindo $41 \%$ dos homens e $39,8 \%$ das mulheres. No ano de 2006, o Ministério da Saúde (MS) desenvolveu o estudo sobre Vigilância de Fatores de Risco e Proteção para Doenças Crônicas por Inquérito Telefônico (VIGITEL), confirmando a tendência de crescimento do excesso de peso no país. Em 2008, esse problema atingia $43,3 \%$ dos adultos, sendo da ordem de $47,3 \%$ nos homens e $39,5 \%$ nas mulheres (RODRIGUES; POMBO; KOIFMAN, 2011).

Uma tendência muito similar vem sendo observada na população infantil em nosso país. De acordo com o ENDEF, realizado em 1975, a prevalência de excesso de peso na faixa etária de dez a 19 anos era de 7,5\% nas meninas e de 3,9\% nos meninos. Na Pesquisa Nacional sobre Saúde e Nutrição (PNSN) desenvolvida em 1989 observou-se um aumento para 13,2 e $8,3 \%$, respectivamente. Após um intervalo de 14 anos, em 2003, a POF apresentou um crescimento mostrando estes índices na casa dos $18 \%$ para o sexo masculino e $15,4 \%$ para o sexo feminino (RODRIGUES; POMBO; KOIFMAN, 2011).

Considerando esse significativo aumento da prevalência de sobrepeso e obesidade em crianças e adolescentes no Brasil e suas consequências, torna-se importante conhecer

DOI: https://doi.org/10.25110/arqsaude.v18i3.2014.5195

${ }^{1}$ Universidade Estadual do Oeste do Paraná - UNIOESTE - ehobold@hotmail.com

${ }^{2}$ Universidade Estadual de Campinas - UNICAMP - miguela@fef.unicamp.br 
a dimensão atual deste problema, realizando um levantamento de estudos que o investiguem identificando quais os critérios utilizados para esta classificação. Assim sendo, o objetivo deste estudo foi revisar as pesquisas de prevalência de sobrepeso e obesidade em crianças e adolescentes brasileiros realizados na última década.

\section{Desenvolvimento}

Na primeira etapa desta revisão sistemática foi utilizada a busca eletrônica de artigos nas bases de dados $S c i E$ LO, PubMed, MedLine e LILACS e na Biblioteca Eletrônica Bireme. Foram utilizados os unitermos: prevalência, sobrepeso, obesidade, excesso de peso, adiposidade, índice de massa corporal, crianças, adolescentes, jovens, escolares. $\mathrm{O}$ período de busca se restringiu a publicações de 2003 a 2013. Os artigos completos foram obtidos por meio de revistas com acesso livre e gratuito.

Foi desenvolvido um protocolo pelos pesquisadores para selecionar os resumos e posteriormente realizar a inclusão e exclusão dos artigos que compõem este estudo. Como critério de inclusão, foi considerada a apresentação de informações relativas à prevalência de sobrepeso e obesidade em crianças e adolescentes brasileiros e o critério de classificação adotado pelo estudo.

Foram excluídos: artigos de revisão, comunicações breves, programas de intervenção, estudos realizados fora do período estabelecido, estudos com estrangeiros e estudos em que a amostra selecionada não fosse representativa do segmento populacional considerado.

Uma ficha para extração de informações dos artigos foi elaborada, na qual foram incluídas informações básicas como nome dos autores, ano de publicação, local de desenvolvimento da pesquisa, tamanho da amostra, faixa etária, sexo, valores relativos de sobrepeso e obesidade e critério de classificação.

Com a utilização dos descritores estabelecidos foram identificados na primeira etapa 793 estudos desenvolvidos sobre sobrepeso e obesidade no Brasil publicados no período de 2003 à 2013. Na segunda etapa verificou-se a relação dos títulos dos artigos com o objetivo proposto reduzindo para 127 artigos. A terceira etapa foi a leitura dos resumos desses artigos. Considerando os critérios de inclusão e exclusão, foram selecionados, após a leitura dos resumos, 82 artigos para leitura na íntegra. A quarta etapa foi a leitura completa dos artigos, sendo que foram excluídos 23 por não fornecerem as informações desejadas.

Após este processo de seleção chegou-se por consenso a um total de 59 artigos para comporem esta revisão sistemática.
A Figura 1 apresenta o fluxograma com as diferentes etapas adotadas para a identificação e inclusão dos estudos.

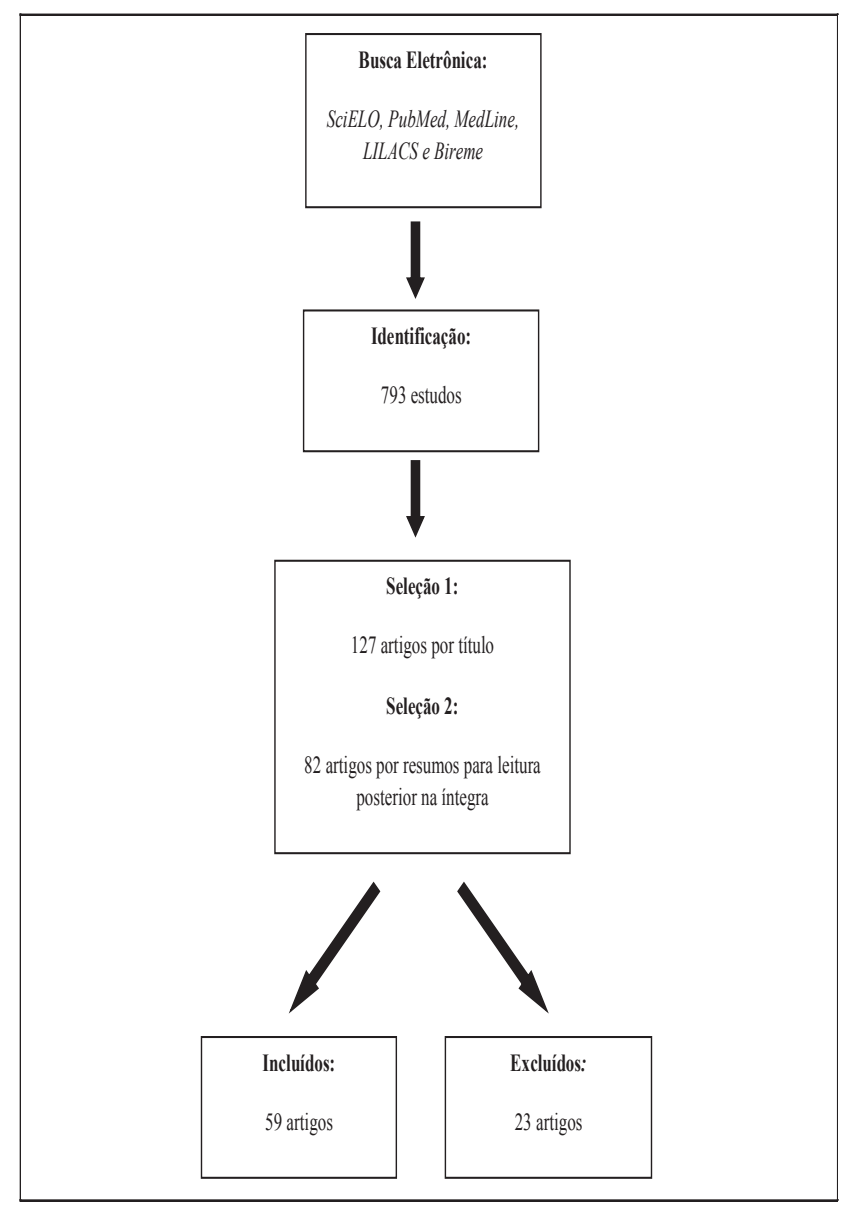

Figura 1: Representação sistemática do método de busca e dos resultados obtidos

Os artigos analisados foram resultados de pesquisas produzidas nas cinco regiões do país (Tabela 1). Região Sul (39\%; n=23); Região Sudeste (32,2\%; n=19); Região Nordeste $(18,6 \% ; n=11)$; Região Norte $(3,4 \% ; n=2)$; Região Centro-oeste $(3,4 \% ; n=2)$; Regiões Nordeste e Sudeste conjuntamente $(1,7 \% ; n=1)$ e todas as regiões do país $(1,7 \%$; $\mathrm{n}=1)$.

Estes envolveram a faixa etária de dois anos (ABRANTES; LAMOUNIER; COLOSIMO, 2003) a vinte anos de idade (GRIZ et al., 2010; PETRIBU et al., 2011) e o tamanho das amostras oscilou de 328 sujeitos (VASCONCELLOS; ANJOS; VASCONCELLOS, 2013) a 79.795 sujeitos (SILVA, 2011).

Tabela 1: Estudos sobre Prevalência de Sobrepeso (SP) e

Obesidade (OB) em crianças e adolescentes brasileiros, revisão sistemática da literatura, 2003-2013.

\begin{tabular}{l|l|l|l|l|l}
\hline Autores/ano & Local & Região & $\mathbf{n}$ & Idade & Critério de Classif. \\
\hline Neves et al. (2006) & Belém PA & Norte & 637 & 6 a 9 anos & Escore Z OMS 1995 \\
\hline Silva-Junior et al. (2012) & Rio Branco AC & Norte & 741 & 14 a 18 anos & IMC OMS 1995 \\
\hline Araújo et al. (2010) & Fortaleza CE & Nordeste & 794 & 12 a 17 anos & IMC Cole et al. 2000 \\
\hline Campos, Leite e Almeida (2006) & Fortaleza CE & Nordeste & 1.158 & 10 a 19 anos & IMC OMS 1995 \\
\hline Griz et al. (2010) & Recife PE & Nordeste & 1.824 & 14 a 20 anos & IMC CDC 2002 \\
\hline Leal et al. (2012) & Recife PE & Nordeste & 1.435 & 5 a 19 anos & Escore Z OMS 1995 \\
\hline
\end{tabular}




\begin{tabular}{|c|c|c|c|c|c|}
\hline Mendonça et al. (2010) & Maceió AL & Nordeste & 1.253 & 7 a 17 anos & IMC CDC 2002 \\
\hline Nunes, Figueiroa e Alves (2007) & C. Grande PB & Nordeste & 588 & 10 a 19 anos & IMC CDC 2002 \\
\hline $\begin{array}{l}\text { Oliveira, Cerqueira e Oliveira } \\
(2003)\end{array}$ & F. Santana BA & Nordeste & 699 & 5 a 9 anos & IMC Cole et al. 2000 \\
\hline Petribu et al. (2011) & Caruaru PE & Nordeste & 600 & 15 a 20 anos & IMC Cole et al. 2000 \\
\hline Silva (2011) & SE & Nordeste & 79.795 & 5 a 10 anos & Escore Z OMS 2005 \\
\hline Silva, Balaban e Mota (2005) & Recife PE & Nordeste & 1.616 & 7 a 19 anos & IMC CDC 2002 \\
\hline Tassitano et al. (2009) & 44 Municíp. PE & Nordeste & 4.210 & 14 a 19 anos & IMC Cole et al. 2000 \\
\hline Baruki et al. (2006) & Corumbá MS & Centro-oeste & 403 & 7 a 19 anos & IMC CDC 2002 \\
\hline Giugliano e Melo (2004) & Brasília DF & Centro-oeste & 528 & 6 a 10 anos & IMC Cole et al. 2000 \\
\hline Anjos et al. (2003) & $\begin{array}{l}\text { Rio de Janeiro } \\
\text { - RJ }\end{array}$ & Sudeste & 3.387 & 4 a 17 anos & $\begin{array}{l}\text { Escore Z e IMC OMS } \\
1995\end{array}$ \\
\hline Branco, Hilário e Cintra (2006) & São Paulo SP & Sudeste & 1.009 & 14 a 19 anos & IMC OMS 1995 \\
\hline Costa, Cintra e Fisberg (2006) & Santos SP & Sudeste & 10.822 & 7 a 10 anos & IMC CDC 2002 \\
\hline Fernandes et al. (2007) & P. Prudente SP & Sudeste & 1.215 & 10 a 17 anos & IMC Cole et al. 2000 \\
\hline Ferreira et al. (2008) & Taguatinga MG & Sudeste & 1550 & 7 a 11 anos & IMC CDC 2002 \\
\hline Freitas-Junior (2008) & P. PrudenteSP & Sudeste & 1.327 & 7 a 19 anos & IMC Cole et al. 2000 \\
\hline Guedes e Mendes (2012) & V. equitinh.MG & Sudeste & 5.100 & 6 a 18 anos & IMC Cole et al. 2000 \\
\hline Guedes et al. (2010) & M. Claros MG & Sudeste & 2.849 & 6 a 18 anos & IMC Cole et al. 2000 \\
\hline $\begin{array}{l}\text { Martins, Ribeiro e Barros-Filho } \\
(2010)\end{array}$ & Sorocaba SP & Sudeste & 11.290 & 7 a 10 anos & IMC CDC 2002 \\
\hline Mondini et al. (2007) & Cajamar SP & Sudeste & 1.014 & 6 e 7 anos & IMC Cole et al. 2000 \\
\hline Nobre et al. (2006) & São Paulo SP & Sudeste & 2.125 & 11 a 14 anos & IMC Must et al. 1991 \\
\hline Pereira et al. (2009) & Itapetininga $\mathrm{MG}$ & Sudeste & 494 & 2 a 19 anos & IMC CDC 2002 \\
\hline Ramos e Barros-Filho (2003) & B. Paulista SP & Sudeste & 1.334 & 11 a 18 anos & IMCMust et al. 1991 \\
\hline Rodrigues et al. (2011) & Juiz de Fora MG & Sudeste & 480 & 4 a 18 anos & $\begin{array}{l}\text { IMC Cole et al. } 2000 \\
\text { OMS } 1995\end{array}$ \\
\hline Ribeiro et al. (2006) & B Horizonte MG & Sudeste & 1.445 & 6 a 18 anos & IMC CDC 1999 \\
\hline $\begin{array}{l}\text { Sotelo, Colugnati e Taddei } \\
\text { (2004) }\end{array}$ & São Paulo SP & Sudeste & 2.509 & 6 a 11 anos & $\begin{array}{l}\text { IMC Cole et al. } 2000 \\
\text { Must et al. } 1991 \text { OMS } \\
1995\end{array}$ \\
\hline Toral, Slater e Silva (2007) & Piracicaba SP & Sudeste & 390 & 10 a 17 anos & IMC CDC 2002 \\
\hline $\begin{array}{l}\text { Vasconcellos, Anjos e } \\
\text { Vasconcellos (2013) }\end{array}$ & Niterói RJ & Sudeste & 328 & 10 a 18 anos & IMCOMS 1995 \\
\hline Vanzelli et al. (2008) & Jundiaí SP & Sudeste & 662 & 10 a 18 anos & $\begin{array}{l}\text { IMC Cole et al. } 2000 \\
\text { Must et al. } 1991\end{array}$ \\
\hline Adami e Vasconcelos (2008) & Florianópolis SC & Sul & 629 & 10 a 14 anos & $\begin{array}{l}\text { IMC Conde e Monteiro } \\
2006\end{array}$ \\
\hline Bernardo et al. (2010) & Florianópolis SC & Sul & 886 & 7 a 14 anos & IMC OMS 1995 \\
\hline Bernardo e Vasconcelos (2012) & Florianópolis SC & Sul & 2.863 & 7 a 14 anos & IMC Cole et al. 2000 \\
\hline Burgos et al. (2010) & S. C. do Sul RS & Sul & 1.666 & 7 a 17 anos & $\begin{array}{l}\text { IMC Conde e Monteiro } \\
2006\end{array}$ \\
\hline Corso et al. (2012) & 8 Municípios SC & Sul & 4.964 & 6 a 10 anos & IMC Cole et al. 2000 \\
\hline Cureau et al. (2012) & Santa Maria RS & Sul & 424 & 14 a 18 anos & $\begin{array}{l}\text { IMC Conde e Monteiro } \\
2006\end{array}$ \\
\hline Dumith, e Farias-Junior (2010) & Rio Grande RS & Sul & 525 & 7 a 15 anos & $\begin{array}{l}\text { IMC Cole et al. } 2000 \\
\text { Conde e Monteiro } 2006 \\
\text { OMS } 1995\end{array}$ \\
\hline Farias-Junior e Lopes (2003) & Florianópolis SC & Sul & 1832 & 15 a 18 anos & IMC Cole et al. 2000 \\
\hline Guedes et al. (2006) & Apucarana PR & Sul & 4.319 & 7 a 18 anos & IMC Cole et al. 2000 \\
\hline Kunkel, Oliveira e Peres (2009) & Florianópolis SC & Sul & 467 & 15 a 18 anos & IMC Cole et al. 2000 \\
\hline
\end{tabular}




\begin{tabular}{|c|c|c|c|c|c|}
\hline Mello et al. (2010) & Marialva PR & Sul & 356 & 6 a 10 anos & IMC Cole et al. 2000 \\
\hline Monteiro e Matioli (2010) & Maringá PR & Sul & 652 & 7 a 10 anos & IMC Must et al. 1991 \\
\hline Netto-Oliveira et al. (2010) & Maringá PR & Sul & 1.634 & 6 a 7,9 anos & IMC Cole et al. 2000 \\
\hline Pelegrini e Petroski (2007) & Florianópolis SC & Sul & 653 & 14 a 18 anos & IMC Cole et al. 2000 \\
\hline Rech et al. (2010) & C. Serrana RS & Sul & 1.442 & 7 a 12 anos & IMC Cole et al. 2000 \\
\hline Ronque et al. (2005) & Londrina PR & Sul & 511 & 7 a 10 anos & IMC CDC 2002 \\
\hline Rosaneli et al. (2012) & Maringá PR & Sul & 5.033 & 6 a10,9 anos & IMC Cole et al. 2000 \\
\hline Salomons, Rech e Loch (2007) & Arapoti PR & Sul & 1.647 & 6 a 10 anos & P/I, P/E, E/IOMS 1977 \\
\hline Silva et al. (2008) & Florianópolis SC & Sul & 1.362 & 7 a 15 anos & IMC Cole et al. 2000 \\
\hline Soar et al. (2004) & Florianópolis SC & Sul & 419 & 7 a 9 anos & IMC Cole et al. 2000 \\
\hline Suñé et al. (2007) & C. da Canoa RS & Sul & 719 & 11 a 13 anos & IMC Cole et al. 2000 \\
\hline Terres et al. (2006) & Pelotas RS & Sul & 960 & 15 a 18 anos & IMC Cole et al. 2000 \\
\hline Vieira et al. (2007) & Pelotas RS & Sul & 4.452 & 10 a 12 anos & $\begin{array}{l}\text { IMC OMS } 1995 \\
\text { Cole et al. } 2000 \\
\end{array}$ \\
\hline $\begin{array}{l}\text { Abrantes, Lamounier e Colosimo } \\
\text { (2003) }\end{array}$ & \multicolumn{2}{|c|}{ Nordeste e Sudeste Brasil } & 6.626 & 2 a 19 anos & IMC Cole et al. 2000 \\
\hline Pelegrini et al. (2010) & \multicolumn{2}{|l|}{5 Regiões Brasil } & 2.913 & 7 a 9 anos & IMC Cole et al. 2000 \\
\hline
\end{tabular}

O Índice de Massa Corporal (IMC) tem sido amplamente utilizado em estudos desta natureza em todo o mundo. Na presente revisão observa-se que $93,2 \%$ das pesquisas utilizaram este indicador, totalizando 55 estudos. O Escore Z foi utilizado em quatro estudos e o protocolo de Peso/Idade, Peso/Estatura e Estatura/Idade em um estudo.

O critério adotado para a classificação do sobrepeso e obesidade na infância e adolescência é um ponto importante e tem causado discussões e análises. Alguns estudos como os de Rodrigues et al. (2011), Sotelo; Colugnati e Taddei (2004), Vanzelli et al. (2008), Dumith e Farias-Junior (2010) e Vieira et al. (2007) foram desenvolvidos no Brasil, visando a discutir este assunto. Os resultados desses estudos mostraram que existem divergências, não havendo similaridades nas classificações observadas quando comparados dois ou mais critérios diferentes de classificação do sobrepeso e obesidade.

Das pesquisas levantadas no presente estudo, o critério de classificação mais utilizado foi o recomendado pela International Obesity Task Force - IOTF (COLE et al., 2000) sendo utilizado em 31 pesquisas. Os critérios sugeridos pela Organização Mundial da Saúde - OMS (WORLD HEALTH ORGANIZATION, 1995) foram utilizados em 14 estudos. Doze pesquisas optaram por utilizar a classificação do Center for Disease Control and Prevention - CDC (CENTER FOR DISEASE CONTROL AND PREVENTION, 2002). A classificação de Must, Dallal e Dietz (1991) foi utilizada em cinco pesquisas e o critério de classificação elaborado especificamente para a população brasileira por Conde e Monteiro (2006) esteve presente em quatro trabalhos.

As magnitudes do sobrepeso e obesidade podem ser observadas na Tabela 2.

Tabela 2: Valores relativos da Prevalência de Sobrepeso (SP) e Obesidade (OB) em crianças e adolescentes brasileiros, revisão sistemática da literatura, 2003-2013.

\begin{tabular}{l|c|c|c|c|c|c|c|c|c}
\hline \multicolumn{1}{c|}{ Autores/ano } & SP & O & + & OB & O & q & SP/OB & ○ & + \\
\hline Neves et al. (2006) & $3 \%$ & $*$ & $*$ & $4,4 \%$ & $*$ & $*$ & $7,4 \%$ & $*$ & $*$ \\
\hline Silva-Junior et al. (2012) & $*$ & $*$ & $*$ & $*$ & $*$ & $*$ & $29,5 \%$ & $33.2 \%$ & $26,4 \%$ \\
\hline Araújo et al. (2010) & $20 \%$ & $22,1 \%$ & $18,5 \%$ & $4 \%$ & $5,4 \%$ & $3,7 \%$ & $24 \%$ & 27,5 & $22,2 \%$ \\
\hline Campos, Leite e Almeida (2006) & $*$ & $*$ & $*$ & $*$ & $*$ & $*$ & $21,1 \%$ & $24,2 \%$ & $17,9 \%$ \\
\hline Griz et al. (2010) & $6,7 \%$ & $*$ & $*$ & $3,7 \%$ & $*$ & $*$ & $10,4 \%$ & $*$ & $*$ \\
\hline Leal et al. (2012) & $9,5 \%$ & $*$ & $*$ & $3,8 \%$ & $*$ & $*$ & $13,3 \%$ & $*$ & $*$ \\
\hline Mendonça et al. (2010) & $9,3 \%$ & $*$ & $*$ & $4,5 \%$ & $*$ & $*$ & $13,8 \%$ & $*$ & $*$ \\
\hline Nunes, Figueiroa e Alves (2007) & $18,3 \%$ & $*$ & $*$ & $6,8 \%$ & $*$ & $*$ & $25,1 \%$ & $*$ & $*$ \\
\hline Oliveira, Cerqueira e Oliveira (2003) & $9,3 \%$ & $*$ & $*$ & $4,4 \%$ & $*$ & $*$ & $13,7 \%$ & $*$ & $*$ \\
\hline Petribú et al. (2011) & $19 \%$ & $16,4 \%$ & $20,6 \%$ & $5,5 \%$ & $6,2 \%$ & $5,1 \%$ & $24,5 \%$ & $22,6 \%$ & $25,7 \%$ \\
\hline Silva (2011) & $12,9 \%$ & $13,0 \%$ & $12,8 \%$ & $12,9 \%$ & $14,4 \%$ & $11,4 \%$ & $25,8 \%$ & $27,4 \%$ & $24,2 \%$ \\
\hline Silva, Balaban e Motta (2005) & $14,5 \%$ & $*$ & $*$ & $8,3 \%$ & $*$ & $*$ & $22,8 \%$ & $*$ & $*$ \\
\hline Tassitano et al. (2009) & $11,5 \%$ & $11,5 \%$ & $11,5 \%$ & $2,4 \%$ & $1,9 \%$ & $2,8 \%$ & $13,9 \%$ & $13,4 \%$ & $14,3 \%$ \\
\hline Baruki et al. (2006) & $6,2 \%$ & $5,0 \%$ & $7,4 \%$ & $6,5 \%$ & $5,3 \%$ & $7,7 \%$ & $12,7 \%$ & $10,3 \%$ & $15,1 \%$ \\
\hline Giugliano e Melo (2004) & $14,6 \%$ & $12,9 \%$ & $16,1 \%$ & $5,5 \%$ & $5,9 \%$ & $5,1 \%$ & $20,1 \%$ & $18,8 \%$ & 21,2 \\
\hline
\end{tabular}




\begin{tabular}{|c|c|c|c|c|c|c|c|c|c|}
\hline Anjos et al. (2003) & $16 \%$ & $14,1 \%$ & $17,7 \%$ & $4,9 \%$ & $4,8 \%$ & $5 \%$ & $20,9 \%$ & $18,9 \%$ & $22,7 \%$ \\
\hline Branco, Hilário e Cintra (2006) & $13,6 \%$ & $15,9 \%$ & $10,2 \%$ & $4,9 \%$ & $5,9 \%$ & $3,4 \%$ & $18,5 \%$ & $21,8 \%$ & $13,6 \%$ \\
\hline Costa, Cintra e Fisberg (2006) & $15,7 \%$ & $*$ & $*$ & $18 \%$ & $*$ & $*$ & $33,7 \%$ & $*$ & $*$ \\
\hline Fernandes et al. (2007) & $*$ & $*$ & $*$ & $*$ & $*$ & $*$ & $28,6 \%$ & $35,7 \%$ & $20 \%$ \\
\hline Ferreira et al. (2008) & $12,6 \%$ & $12,8 \%$ & $12,4 \%$ & $8 \%$ & $10,2 \%$ & $5,8 \%$ & $20,6 \%$ & $23 \%$ & $18,2 \%$ \\
\hline Freitas-Junior (2008) & $15 \%$ & $13,7 \%$ & $16,4 \%$ & $8,4 \%$ & $11,4 \%$ & $5,3 \%$ & $23,4 \%$ & $25 \%$ & $21,6 \%$ \\
\hline Guedes e Mendes (2012) & $10 \%$ & $6,6 \%$ & $13,5 \%$ & $1,3 \%$ & $1,3 \%$ & $1,3 \%$ & $11,3 \%$ & $7,9 \%$ & $14,8 \%$ \\
\hline Guedes et al. (2010) & $17,2 \%$ & $14,7 \%$ & $19,7 \%$ & $3,8 \%$ & $2,8 \%$ & $4,8 \%$ & $21 \%$ & $17,5 \%$ & $24,5 \%$ \\
\hline Martins Ribeiro e Barros-F & $12,2 \%$ & $12,1 \%$ & $12,3 \%$ & $10,1 \%$ & $11,9 \%$ & $8,2 \%$ & $22,3 \%$ & $24 \%$ & $20,5 \%$ \\
\hline Mondini et al. (2007) & $10,8 \%$ & $*$ & $*$ & $6,2 \%$ & $*$ & $*$ & $17 \%$ & $*$ & $*$ \\
\hline Nobre et al. (2006) & $*$ & $*$ & $*$ & $*$ & $*$ & $*$ & $24 \%$ & $*$ & $*$ \\
\hline Pereira et al. (2009) & $9,6 \%$ & $8,6 \%$ & $10,5 \%$ & $13,8 \%$ & $19,2 \%$ & 8,4 & $23,4 \%$ & $27,8 \%$ & $18,9 \%$ \\
\hline Ramos e Barros Filhos (2 & $7,3 \%$ & $8,9 \%$ & $6,2 \%$ & $3,7 \%$ & $3,9 \%$ & $3,3 \%$ & $11 \%$ & $12,8 \%$ & $9,5 \%$ \\
\hline Ribeiro et al. (2006) & $8,4 \%$ & $*$ & $*$ & $3,1 \%$ & $*$ & $*$ & $11,5 \%$ & $*$ & $*$ \\
\hline \multirow{2}{*}{ Rodrigues et al. (2011) } & $12,2 \%$ & $11,8 \%$ & $12,5 \%$ & $3,6 \%$ & $2,9 \%$ & $4,2 \%$ & $15,8 \%$ & $14,7 \%$ & $16,7 \%$ \\
\hline & $10,3 \%$ & $13,1 \%$ & $7,5 \%$ & $9 \%$ & $7,6 \%$ & 10,4 & $19,3 \%$ & $20,7 \%$ & $17,9 \%$ \\
\hline \multirow{3}{*}{ Sotelo, Colugnati e Taddei (2004) } & $12,2 \%$ & $10,9 \%$ & $13,4 \%$ & $8,2 \%$ & $8,2 \%$ & $8,2 \%$ & $20,4 \%$ & $19,1 \%$ & $21,6 \%$ \\
\hline & $12,1 \%$ & $10,2 \%$ & $13,9 \%$ & $15,1 \%$ & $13,7 \%$ & $16,5 \%$ & $27,2 \%$ & $23,9 \%$ & $30,4 \%$ \\
\hline & $12,8 \%$ & $11,9 \%$ & $13,7 \%$ & $11 \%$ & $10,3 \%$ & $11,7 \%$ & $23,8 \%$ & $22,2 \%$ & $25,4 \%$ \\
\hline Toral, Slater e Silva (2007) & $10,3 \%$ & $10 \%$ & 10,5 & $10,9 \%$ & $13,8 \%$ & $8,1 \%$ & $21,2 \%$ & $23,8 \%$ & $18,6 \%$ \\
\hline $\begin{array}{l}\text { Vasconcellos, Anjos e Vasconcellos } \\
\text { (2013) }\end{array}$ & $18 \%$ & $*$ & $*$ & $7,7 \%$ & $*$ & $*$ & $25,7 \%$ & $24,5 \%$ & $26,3 \%$ \\
\hline \multirow{2}{*}{ Vanzelli et al. (2008) } & $16 \%$ & $14 \%$ & $18 \%$ & $8 \%$ & $8 \%$ & $8 \%$ & $24 \%$ & $22 \%$ & $26 \%$ \\
\hline & $13 \%$ & $12 \%$ & $14 \%$ & $9 \%$ & $9 \%$ & $9 \%$ & $22 \%$ & $21 \%$ & $23 \%$ \\
\hline Adami e Vasconcelos (2008) & $22,2 \%$ & $27,9 \%$ & $16,5 \%$ & $5,5 \%$ & $5,8 \%$ & $5,1 \%$ & $27,7 \%$ & $33,7 \%$ & $21,6 \%$ \\
\hline Bernardo et al. (2010) & $13,9 \%$ & $17,3 \%$ & $10,6 \%$ & $9,9 \%$ & $12,7 \%$ & $7,1 \%$ & $23,8 \%$ & $20 \%$ & $17,7 \%$ \\
\hline Bernardo e Vasconcelos (2012) & $*$ & $*$ & $*$ & $*$ & $*$ & $*$ & $21,9 \%$ & $25,3 \%$ & $18,6 \%$ \\
\hline Burgos et al. (2010) & $19 \%$ & $21 \%$ & $16,9 \%$ & $7,7 \%$ & $6,1 \%$ & $9,4 \%$ & $26,7 \%$ & $27,1 \%$ & $26,3 \%$ \\
\hline Corso et al. (2012) & $15,4 \%$ & $15,4 \%$ & $15,4 \%$ & $6,1 \%$ & $6,1 \%$ & $6,1 \%$ & $21,5 \%$ & $21,5 \%$ & $21,5 \%$ \\
\hline & $19,6 \%$ & $*$ & $*$ & $4,2 \%$ & $*$ & $*$ & $23,8 \%$ & $32,5 \%$ & $15,1 \%$ \\
\hline \multirow{3}{*}{ Dumith e Farias-Junior (2010) } & $21 \%$ & $19,6 \%$ & $22,4 \%$ & $7,4 \%$ & $7,7 \%$ & $7,2 \%$ & $28,4 \%$ & $27,3 \%$ & $29,6 \%$ \\
\hline & $26,5 \%$ & $26,1 \%$ & $26,9 \%$ & $9,5 \%$ & $6,9 \%$ & $12,1 \%$ & $36 \%$ & $33 \%$ & $39 \%$ \\
\hline & $25 \%$ & $25,5 \%$ & $24,5 \%$ & $11,5 \%$ & $12,7 \%$ & $10,4 \%$ & $36,5 \%$ & $38,2 \%$ & $34,9 \%$ \\
\hline Farias-Junior e Lopes (2003) & $*$ & $*$ & $*$ & $*$ & $*$ & $*$ & $11,4 \%$ & $14,8 \%$ & $8 \%$ \\
\hline Guedes et al. (2006) & $23,3 \%$ & $21,9 \%$ & $24,7 \%$ & $5 \%$ & $4,1 \%$ & $5,9 \%$ & $28,3 \%$ & $26 \%$ & $30,6 \%$ \\
\hline Kunkel, Oliveira e Peres (2009) & $12,2 \%$ & $17,9 \%$ & $8,8 \%$ & $3,6 \%$ & $2,3 \%$ & $4,4 \%$ & $15,8 \%$ & $20,2 \%$ & $13,2 \%$ \\
\hline Mello et al. (2010) & $20,2 \%$ & $*$ & $*$ & $7 \%$ & $*$ & $*$ & $27,2 \%$ & $27,6 \%$ & $26,9 \%$ \\
\hline Monteiro e Matioli (2010) & $21,9 \%$ & $10,1 \%$ & 11,8 & $13,3 \%$ & $7,8 \%$ & $5,5 \%$ & $35,2 \%$ & $17,9 \%$ & $17,3 \%$ \\
\hline Netto-Oliveira et al. (2010) & $13,8 \%$ & $6,7 \%$ & $7,1 \%$ & $8,3 \%$ & $4,3 \%$ & $4 \%$ & $22,1 \%$ & $11 \%$ & $11,1 \%$ \\
\hline Pelegrini e Petroski (2007) & $10,2 \%$ & $10,6 \%$ & $9,8 \%$ & $3,6 \%$ & $3 \%$ & $4,3 \%$ & $13,8 \%$ & $13,6 \%$ & $14,1 \%$ \\
\hline Rech et al. (2010) & $19,9 \%$ & $19,4 \%$ & $20,4 \%$ & $8 \%$ & $8,7 \%$ & $7,4 \%$ & $27,9 \%$ & $28,1 \%$ & $27,8 \%$ \\
\hline Ronque et al. (2005) & $18,5 \%$ & $19,7 \%$ & $17,3 \%$ & $13,4 \%$ & $17,5 \%$ & $9,3 \%$ & $31,9 \%$ & $37,2 \%$ & $26,6 \%$ \\
\hline Rosanelli et al. (2012) & $17 \%$ & $*$ & $*$ & $7 \%$ & $*$ & $*$ & $26,4 \%$ & $28 \%$ & $24,7 \%$ \\
\hline Salomons, Rech e Loch (2007) & $10 \%$ & $9,4 \%$ & $10,6 \%$ & $10,9 \%$ & $10,1 \%$ & $11,7 \%$ & $20,9 \%$ & $19,5 \%$ & $22,3 \%$ \\
\hline Silva et al. (2008) & $*$ & $*$ & $*$ & $*$ & $*$ & $*$ & $15,6 \%$ & $16 \%$ & $12,6 \%$ \\
\hline Soar et al. (2004) & $17,9 \%$ & $19,1 \%$ & $16,7 \%$ & $6,7 \%$ & $7,9 \%$ & $5,4 \%$ & $24,6 \%$ & $27 \%$ & $22,1 \%$ \\
\hline Suñé et al. (2007) & $21,3 \%$ & $*$ & $*$ & $3,5 \%$ & $*$ & $*$ & $24,8 \%$ & $27,9 \%$ & $21,7 \%$ \\
\hline Terres et al. (2006) & $20,9 \%$ & $21,3 \%$ & $20,5 \%$ & $5 \%$ & $5,1 \%$ & $4,8 \%$ & $25,9 \%$ & $26,4 \%$ & $25,3 \%$ \\
\hline
\end{tabular}




\begin{tabular}{l|c|c|c|c|c|c|c|c|c}
\hline & $23,2 \%$ & $25,1 \%$ & $21,3 \%$ & $11,6 \%$ & $15,1 \%$ & $8,2 \%$ & $34,8 \%$ & $40,2 \%$ & $29,5 \%$ \\
\cline { 2 - 8 } Vieira et al. (2007) & $21,6 \%$ & $22,3 \%$ & $20,9 \%$ & $5 \%$ & $5,6 \%$ & $4,5 \%$ & $26,6 \%$ & $27,9 \%$ & $25,4 \%$ \\
\hline $\begin{array}{l}\text { Abrantes, Lamounier e Colosimo } \\
(2003)\end{array}$ & $15,8 \%$ & $9,4 \%$ & $22,2 \%$ & $9,1 \%$ & $8,6 \%$ & $9,6 \%$ & $24,9 \%$ & $18 \%$ & $31,8 \%$ \\
\hline Pelegrini et al. (2010) & $15,4 \%$ & $15,9 \%$ & $15 \%$ & $7,8 \%$ & $8 \%$ & $7,5 \%$ & $23,2 \%$ & $23,9 \%$ & $22,5 \%$ \\
\hline
\end{tabular}

*Valores não Informados nos artigos.

A menor prevalência de sobrepeso encontrada foi de 3\% (NEVES et al., 2006), de obesidade foi de 1,3\% (GUEDES; MENDES, 2012) e da somatória do sobrepeso e da obesidade foi de 7,4\% (NEVES et al., 2006). As maiores prevalências foram de 26,5\% (DUMITH; FARIAS-JUNIOR, 2010), 15,1\% (SOTELO; COLUGNATI; TADDEI, 2004) e 36,5\% (DUMITH; FARIAS-JUNIOR, 2010), respectivamente.

No sexo masculino a prevalência mínima de sobrepeso foi de 5\% (BARUKI et al., 2006) e máxima de 27,9\% (ADAMI; VASCONCELOS, 2008). No feminino foram respectivamente de $6,2 \%$ (RAMOS; BARROS FILHO, 2003) e 26,9\% (DUMITH; FARIAS-JUNIOR, 2010). Não foram observadas diferenças estatísticas no sobrepeso entre os sexos $(\mathrm{t}=-0,210 ; \mathrm{p}=0,835)$.

Quanto à obesidade no sexo masculino os valores mínimos e máximos observados foram respectivamente 1,3\% (GUEDES; MENDES, 2012) e 19,2\% (PEREIRA et al., 2009) e no feminino 1,3\% (GUEDES; MENDES, 2012) e 16,5\% (SOTELO; COLUGNATI; TADDEI, 2004). Também não foram observadas diferenças significativas entre os sexos $(\mathrm{t}=1,960 ; \mathrm{p}=0,056)$.

Quanto à prevalência de sobrepeso e obesidade conjuntamente identificou-se diferença significativa entre os sexos $(t=2,264 ; p=0,028)$, sugerindo que o sexo masculino possui uma prevalência maior de sobrepeso e obesidade.

No Brasil, temos observado o significativo crescimento do sobrepeso e da obesidade de crianças e adolescentes e consequentemente de adultos. Rodrigues, Pombo e Koifman (2011) enfatizam os estudos do ENDEF (1975), PNSN (1989), POF (2003) e VIGITEL (2006), sendo que estes estudos mostram que em quase quarenta anos estas disfunções vêm aumentando de forma significativa em nosso país.

Diversos pesquisadores brasileiros, relacionados no presente estudo, vêm observando o comportamento dessas prevalências e apesar de não haver um consenso sobre os critérios de classificação, as pesquisas mostram dados cada vez mais alarmantes. Nesta revisão, observou-se que não existe uma unanimidade nos pontos de corte adotados. Guedes et al. (2010) ressaltam que este é o maior obstáculo para a realização de análises comparativa entre diferentes estudos. Entretanto, isto não nos isenta da preocupação com o contínuo crescimento deste problema no Brasil.

A prevalência de sobrepeso/obesidade nestes estudos oscilou de 7,4\% a 36,5\%. Mais de 70\% dos estudos mostraram valores de prevalência superior a $20 \%$. Um estudo de revisão publicado por Araújo et al. (2007) mostrou uma variação de $4 \%$ a $31 \%$ na prevalência de excesso de peso em adolescentes brasileiros, sendo que, mais de $60 \%$ dos estudos descreveram prevalências superiores a $15 \%$. Comparado com os dados desta revisão vemos o expressivo aumento em um curto período de tempo.

As oscilações nos valores de prevalência de sobre- peso/obesidade podem ser afetadas por diversos fatores, sendo socioeconômicos, culturais, geográficos, metodológicos e de critérios de classificação.

A maioria dos estudos desta revisão foi realizada nas regiões Sul e Sudeste, considerados mais desenvolvidos socioeconomicamente, entretanto não se observa diferenças quando comparado às outras regiões, sugerindo assim, que o problema do sobrepeso e obesidade de crianças e adolescentes afeta o país todo e não apenas algumas regiões específicas.

Quando observamos a prevalência de sobrepeso e obesidade separadamente e por sexo verifica-se um equilíbrio nos valores levantados pelos pesquisadores. Entretanto, quando observada a prevalência de sobrepeso/obesidade conjuntamente é visível que a mesma é mais elevada no sexo masculino na grande maioria dos estudos analisados sistematicamente. Resultados similares foram observados na Índia (RAMACHANDRAN et al., 2002) e nos Estados Unidos (OGDEN et al., 2012).

Como limitação deste estudo reconhece-se que apesar da busca criteriosa realizada pelos pesquisadores, não se descarta a possibilidade de que algum estudo importante sobre a prevalência de sobrepeso/obesidade em crianças e adolescentes tenha ficado fora desta revisão.

Baseado nas pesquisas analisadas, conclui-se que a maioria dos estudos sobre prevalência de sobrepeso/obesidade em crianças e adolescentes foi desenvolvida nas regiões Sul e Sudeste, envolvendo amostras probabilísticas e utilizando diferentes protocolos e diferentes critérios de classificação (pontos de corte). É preocupante a prevalência de sobrepeso/obesidade em crianças e adolescentes brasileiros, considerando que mais de $70 \%$ dos estudos analisados mostraram uma prevalência superior a $20 \%$, sendo mais elevada no sexo masculino e de forma homogênea em todas as regiões do país.

\section{Referências}

ABRANTES, M. M.; LAMOUNIER, J. A.; COLOSIMO, E. A. Prevalência de sobrepeso e obesidade nas regiões nordeste e sudeste do Brasil. Rev Assoc Med Bras, v. 49, n. 2, p. 162-166, 2003.

ADAMI, F.; VASCONCELOS, F. A. G. Obesidade e maturação sexual precoce em escolares de Florianópolis SC. Rev Bras Epidemiol, v. 11, n. 4, p. 549-560, 2008.

ANJOS, L. A. et al. Crescimento e estado nutricional em amostra probabilística de escolares no Município do Rio de Janeiro, 1999. Cad Saúde Pública, v. 19, Suppl.1, p. 171179, 2003.

ARAÚJO, M. F. M. et al. Sobrepeso entre adolescentes de 
escolas particulares de Fortaleza, CE, Brasil. Rev Bras Enferm, v. 63, n. 4, p. 623-628, 2010.

ARAUjO, V. C. et al. Prevalência de excesso de peso em adolescentes brasileiros: um estudo de revisão sistemática. Rev Bras Ativ Fís Saúde, v. 12, n. 3, p. 79-87, 2007.

BARUKI, S. B. S. et al. Associação entre estado nutricional e atividade física em escolares da Rede Municipal de Ensino em Corumbá - MS. Rev Bras Med Esporte, v. 12, n. 2, p. 90-94, 2006.

BERNARDO, C. O. et al. Associação entre o índice de massa corporal de pais e de escolares de 7 a 14 anos de Florianópolis, SC, Brasil. Rev Bras Saúde Matern Infant, v. 10, n. 2, p. 183-190, 2010.

BERNARDO, C. O.; VASCONCELOS, F. A. G. Association of parents' nutritional status, and sociodemographic and dietary factorswith overweight/ obesity in schoolchildren 7 to 14 years old. Cad Saúde Pública, v. 28, n. 2, p. 291-304, 2012.

BRANCO, L. M.; HILÁRIO, M. O. E.; CINTRA, I. P. Percepção e satisfação corporal em adolescentes e a relação com seu estado nutricional. Rev Psiq Clín, v. 33, n. 6, p. 292-296, 2006.

BURGOS, M. Z. et al. Uma análise entre índices pressóricos, obesidade e capacidade cardiorrespiratória em escolares. Arq Bras Cardiol, v. 94, n. 6, p. 6p., 2010.

CAMPOS, L. A.; LEITE, A. J. M.; ALMEIDA, P. C. A. Nível socioeconômico e sua influência sobre a prevalência de sobrepeso e obesidade em escolares adolescentes do município de Fortaleza. Rev Nutr, v. 19, n. 5, p. 531-538, 2006 .

CENTER FOR DISEASE CONTROL AND PREVENTION - CDC. 2002. Disponível em: <http://www.cdc.gov/gshs $>$. Acesso em: 15 ago. 2013.

COLE, T. J. et al. Establishing a standard definition for child overweight and obesity worldwide: international survey. Br Med J, v. 320, p.1240-1243, 2000.

CONDE, W. L.; MONTEIRO, C. A. Brazilian body mass index cut off points for evaluation of nutritional status from children and adolescents. J Pediatr, v. 82, n. 4, p. 266-272, 2006.

CORSO, A. C. T. et al. Fatores comportamentais associados ao sobrepeso e à obesidade em escolares do Estado de Santa Catarina. Rev Bras Est Pop, v. 29, n. 1, p. 117-131, 2012.

COSTA, R. F.; CINTRA, I. P.; FISBERG, M. Prevalência de Sobrepeso e Obesidade em Escolares da Cidade de Santos, SP. Arq Bras Endocrinol Metab, v. 50, n.1, p. 6067, 2006.

CUREAU, F. V. et al. Sobrepeso/obesidade em adolescentes de Santa Maria-RS: prevalência e fatores associados. Rev Bras Cineantropom Desempenho Hum, v. 14, n. 5, p. 517-526, 2012.

DUMITH, S. C.; FARIAS-JUNIOR, J. C. Sobrepeso e obesidade em crianças e adolescentes: comparação de três critérios de classificação baseados no índice de massa corporal. Rev Panam Salud Publica, v. 28, n. 1, p. 30-35, 2010 .

FARIAS-JUNIOR, J. C.; LOPES, A. S. Prevalência de sobrepeso em adolescentes. Rev Bras Ci e Mov, v. 11, n. 3, p. 77-84, 2003.

FERNANDES, R. A. et al. Prevalência de sobrepeso e obesidade em alunos de escolas privadas do município de Presidente Prudente - SP. Rev Bras Cineantropom Desempenho Hum, v. 9, n. 1, p. 21-27, 2007.

FERREIRA, A. P. et al. Prevalência de sobrepeso e obesidade em escolares de Taguatinga - DF. Rev Inst Ciênc Saúde, v. 26, n. 2, p. 161-166, 2008.

FREITAS-JÚNIOR, I. F. et al. Crescimento e estado nutricional de crianças e adolescentes de Presidente Prudente, São Paulo, Brasil. Rev Bras Saúde Matern Infant, v. 8, n. 3, p. 265-274, 2008.

GIUGLIANO, R.; MELO, A. L. P. Diagnóstico de sobrepeso e obesidade em escolares: utilização do índice de massa corporal segundo padrão internacional. J Pediatria, v. 80, n. 2, p. 129-134, 2004.

GRIZ, L. H. M. et al. Prevalence of central obesity in a large sample of adolescents from public schools in Recife, Brazil. Arq Bras Endocrinol Metab, p. 54-57, 2010.

GUEDES, D. P. et al. Impacto de fatores sociodemográficos e comportamentais na prevalência de sobrepeso e obesidade de escolares. Rev Bras Cineantropom Desempenho Hum, v. 12, n. 4, p. 221-231, 2010.

GUEDES, D. P. et al. Prevalência de sobrepeso e obesidade em crianças e adolescentes: estimativas relacionadas ao sexo, à idade e a classe socioeconômica. Rev Bras Educ Fís Esp, v. 20, n. 3, p. 151-163, 2006.

GUEDES, D. P.; MENDES, R. R. Crescimento físico e estado nutricional de escolares do Vale do Jequitinhonha, Minas Gerais, Brasil. Rev Bras Cineantropom Desempenho Hum, v. 14, n. 4, p. 363-376, 2012.

KUNKEL, N.; OLIVEIRA, W. F.; PERES, M. A. Excesso de peso e qualidade de vida relacionada à saúde em adolescentes de Florianópolis, SC. Rev Saúde Pública, v. 43, n. 2, p. 226-235, 2009.

LEAL, V. S. et al. Excesso de peso em crianças e adolescentes no Estado de Pernambuco, Brasil: prevalência e determinantes. Cad Saúde Pública, v. 28, n. 6, p. 1175$1182,2012$. 
MARTINS, C. E.; RIBEIRO, R. R.; BARROS-FILHO, A. A. Estado nutricional de escolares segundo a localização geográfica das escolas em Sorocaba, São Paulo. Rev Paul Pediatr, v. 28, n. 1, p. 55-62, 2010.

MELLO, A. D. M. et al. Prevalência de sobrepeso e obesidade em crianças de seis a dez anos de escolas municipais de área urbana. Rev Paul Pediatr, v. 28, n. 1, p. 48-54, 2010.

MENDONÇA, M. R. T. et al. Prevalência de sobrepeso e obesidade em crianças e adolescentes da cidade de Maceió. Rev Assoc Med Bras, v. 56, n. 2, p. 192-196, 2010.

MONDINI. L. et al. Prevalência de sobrepeso e fatores associados em crianças ingressantes no ensino fundamental em um município da região metropolitana de São Paulo, Brasil. Cad. Saúde Pública, v. 23, n. 8, p. 1825-1834, 2007.

MONTEIRO, V.; MATIOLI, G. Implication of anthropometric profile and alimentary consumption on risk for diseases among school children in the 1st to 4th grades. Brazilian Journal of Pharmaceutical Sciences, v. 46, n. 3, p. 445-454, 2010.

MUST, A.; DALLAL, G. E.; DIETZ, W. H. Reference data for obesity: 85 th and $95^{\text {th }}$ percentiles of body mass index (wt/ht2) and triceps skinfold thickness. Am J Clin Nutr, v. 53, p. 839-846, 1991.

NETTO-OLIVEIRA, E. R. et al. Sobrepeso e obesidade em crianças de diferentes níveis econômicos. Rev Bras Cineantropom Desempenho Hum, v. 12, n. 2, p. 83-89, 2010 .

NEVES, O. M. D. et al. Antropometria de escolares ao ingresso no ensino fundamental na cidade de Belém, Pará, 2001. Rev Bras Saúde Matern Infant, v. 6, n. 1, p. 39-46, 2006.

NOBRE, M. R. C. et al. Prevalências de sobrepeso, obesidade e hábitos de vida associados ao risco cardiovascular em alunos do ensino fundamental. Rev Assoc Med Bras, v. 52, n. 2, p. 118-124, 2006.

NUNES, M. M. A.; FIGUEIROA, J. N.; ALVES, J. G. B. Excesso de peso, atividade física e hábitos alimentares entre adolescentes de diferentes classes econômicas em Campina Grande (PB). Rev Assoc Med Bras, v. 53, n. 2, p. 130-134, 2007.

OGDEN, C. L. et al. Prevalence of Obesity and Trends in Body Mass Index Among US Children and Adolescents, 1999-2010. JAMA, v. 307, n. 5, p. 483-490, 2012.

OLIVEIRA, A. M. A.; CERQUEIRA, E. M. M.; OLIVEIRA, A. C. Prevalência de sobrepeso e obesidade infantil na cidade de Feira de Santana-BA: detecção na família x diagnóstico clínico. J Pediatr, v. 79, n. 4, p. 325$328,2003$.
PELEGRINI, A. et al. Sobrepeso e obesidade em escolares brasileiros de sete a nove anos: dados do projeto Esporte Brasil. Rev Paul Pediatr, v. 28, n. 3, p. 290-295, 2010.

PELEGRINI, A.; PETROSKI, E. L. Excesso de peso em adolescentes: prevalência e fatores associados. Rev Bras Ativ Fís Saúde, v. 12, n. 3, p. 45-53, 2007.

PEREIRA, A. et al. A obesidade e sua associação com os demais fatores de risco cardiovascular em escolares de Itapetininga, Brasil. Arq Bras Cardiol, v. 93, n. 3, p. 253$260,2009$.

PETRIBÚ, M. M. V. et al. Fatores associados ao sobrepeso e à obesidade em estudantes do ensino médio da rede pública estadual do município de Caruaru (PE). Rev Paul Pediatr, v. 29, n. 4, p. 536-545, 2011.

RAMACHANDRAN, A. et al. Prevalence of overweight in urban Indian adolescent school children. Diabetes Research and Clinical Practice, v. 57, n. 3, p. 185-190, 2002.

RAMOS, A. M. P. P.; BARROS-FILHO, A. A. Prevalência da obesidade em adolescentes de Bragança Paulista e sua relação com a obesidade dos pais. Arq Bras Endocrinol Metab, v. 47, n. 6, p. 663-668, 2003.

RECH, R. R. et al. Prevalência de obesidade em escolares de 7 a 12 anos de uma cidade Serrana do RS, Brasil. Rev Bras Cineantropom Desempenho Hum, v. 12, n. 2, p. 90-97, 2010.

RIBEIRO, R. Q. C. et al. Fatores adicionais de risco cardiovascular associados ao excesso de peso em crianças e adolescentes. O estudo do coração de Belo Horizonte. Arq Bras Cardiol, v. 86, n. 6, p. 408-418, 2006.

RODRIGUES, L. G.; POMBO, N.; KOIFMAN, S. Prevalência de alterações metabólicas em crianças e adolescentes com sobrepeso e obesidade: uma revisão sistemática. Rev Paul Pediatr, v. 29, n. 2, p. 277-288, 2011.

RODRIGUES, P. A. et al. Prevalência e fatores associados a sobrepeso e obesidade em escolares da rede pública. Ciên \& Saúde Coletiva, v. 16, Suppl 1, p. S1581-S1588, 2011.

RONQUE, E. R. V. et al. Prevalência de sobrepeso e obesidade em escolares de alto nível socioeconômico em Londrina, Paraná, Brasil. Rev Nutr, v. 18, n. 6, p. 709-717, 2005.

ROSANELI, C. F. et al. Avaliação da prevalência e de determinantes nutricionais e sociais do excesso de peso em uma população de escolares: análise transversal em 5.037 crianças. Rev Assoc Med Bras, v. 58, n. 4, p. 472-476, 2012.

SALOMONS, E.; RECH, C. R.; LOCH, M. R. Estado nutricional de escolares de seis a dez anos de idade da 
rede municipal de ensino de Arapoti, Paraná. Rev Bras Cineantropom Desempenho Hum, v. 9, n. 3, p. 244-249, 2007.

SILVA, D. A. S. Sobrepeso e obesidade em crianças de cinco a dez anos de idade beneficiárias do programa bolsa família no estado de Sergipe, Brasil. Rev Paul Pediatr, v. 29, n. 4, p. 529-535, 2011.

SILVA, G. A. P.; BALABAN, G.; MOTTA, M. E. F. A. Prevalência de sobrepeso e obesidade em crianças e adolescentes de diferentes condições socioeconômicas. Rev Bras Saúde Matern Infant, v. 5, n. 1, p. 53-59, 2005.

SILVA, K. S. et al. Prevalência de excesso de peso corporal em escolas públicas e privadas da cidade de Florianópolis, SC. Arq Bras Endrocrinol Metab, v. 52, n. 3, p. 574-575, 2008.

SILVA-JUNIOR, L. M. et al. Prevalência de excesso de peso e fatores associados em adolescentes de escolas privadas de região urbana na Amazônia. Rev Paul Pediatr, v. 30, n. 2, p. 217-222, 2012.

SOAR, C. et al. Prevalência de sobrepeso e obesidade em escolares de uma escola pública de Florianópolis, Santa Catarina. Rev Bras Saúde Matern Infant, v. 4, n. 4, p. 391-397, 2004.

SOTELO, Y. O. M.; COLUGNATI, F. A. B.; TADDEI, J. A. A. Prevalência de sobrepeso e obesidade entre escolares da rede pública segundo três critérios de diagnóstico antropométrico. Cad Saúde Pública, v. 20, n. 1, p. 233240, 2004.

SUÑÉ, F. R. et al. Prevalência e fatores associados para sobrepeso e obesidade em escolares de uma cidade no Sul do Brasil. Cad Saúde Pública, v. 23, n. 6, p. 1361-1371, 2007.

TASSITANO, R. M. et al. Prevalência e fatores associados ao sobrepeso e à obesidade em adolescentes, estudantes de escolas de ensino médio de Pernambuco, Brasil. Cad Saúde Pública, v. 25, n. 12, p. 2639-2652, 2009.

TERRES, N. G. et al. Prevalência e fatores associados ao sobrepeso e à obesidade em adolescentes. Rev Saúde Pública, v. 40, n. 4, p. 627-633, 2006.

TORAL, N.; SLATER, B.; SILVA, M. V. Consumo alimentar e excesso de peso de adolescentes de Piracicaba, São Paulo. Rev Nutr, v. 20, n. 5, p. 449-459, 2007.

VANZELLI, A. S. et al. Prevalência de sobrepeso e obesidade em escolares da rede pública do município de Jundiaí, São Paulo. Rev Paul Pediatr, v. 26, n. 1, p. 48-53, 2008 .

VASCONCELLOS, M. B.; ANJOS, L. A.; VASCONCELLOS. M. T. L. Estado nutricional e tempo de tela de escolares da rede pública de ensino fundamental de
Niterói, Rio de Janeiro, Brasil. Cad Saúde Pública, v. 29, n. 4, p. 713-722, 2013.

VIEIRA, M. F. A. et al. Diagnosis of overweight and obesity in adolescents from the 1993 Pelotas Birth Cohort Study, Rio Grande do Sul State, Brazil: comparison of two diagnostic criteria. Cad Saúde Pública, v. 23, n. 12, p. 2993-2999, 2007.

WORLD HEALTH ORGANIZATION (WHO). Obesity: preventing and managing the global epidemic. WHO Technical Report Series, no 894. WHO: Geneva; 2000.

WORLD HEALTH ORGANIZATION (WHO). Physical status: the use and interpretation of anthropometry. (Technical Report Series, 854). Geneva: 1995. 